\title{
Correction to: Dissatisfaction After Post-Mastectomy Breast Reconstruction: A Mixed-Methods Study
}

\author{
Ronnie L. Shammas, MD ${ }^{1}\left(\mathbb{D}\right.$, Laura J. Fish, PhD, MPH ${ }^{2,3}$, Amanda R. Sergesketter, MD ${ }^{1}$, \\ Anaeze C. Offodile II, MD, MPH ${ }^{4}$, Brett T. Phillips, MD ${ }^{1}$, Sachi Oshima, BS ${ }^{1}$, Clara N. Lee, MD ${ }^{3}$, \\ Scott T. Hollenbeck, $\mathrm{MD}^{1}$, and Rachel A. Greenup, MD, $\mathrm{MPH}^{5}$
}

${ }^{1}$ Division of Plastic, Maxillofacial, and Oral Surgery, Duke University, Durham, NC; ${ }^{2}$ Cancer Control and Population Sciences, Duke Cancer Institute, Durham, NC; ${ }^{3}$ Department of Plastic and Reconstructive Surgery, College of Medicine, Division of Health Services Management and Policy, College of Public Health, OSU Comprehensive Cancer Center, The Ohio State University, Columbus, OH; ${ }^{4}$ Department of Plastic and Reconstructive Surgery, University of Texas MD Anderson Cancer Center, Houston, TX; ${ }^{5}$ Department of Surgery, Section Chief of Breast Surgery, Yale School of Medicine, DUMC 3513, New Haven, CT

\section{CORRECTION TO: ANN SURG ONCOL HTTPS://D OI.ORG/10.1245/S10434-021-10720-9}

Amanda R. Sergesketter's and Anaeze C. Offodile II's names are correct as reflected here. The original article was corrected.
Publisher's Note Springer Nature remains neutral with regard to jurisdictional claims in published maps and institutional affiliations.

The original article can be found online at https://doi.org/10.1245/ s10434-021-10720-9.

(C) Society of Surgical Oncology 2021

Published Online: 13 September 2021

R. A. Greenup, MD, MPH

e-mail: rachel.greenup@yale.edu 